in Salt Lake City, Utah, conducted a matched case-control study with 1,580 cases and 20,197 controls. $^{2}$ ADEs were found to complicate 2.43 per 100 admissions to the LDS Hospital during the 4-year study period from January 1, 1990, to December 31, 1993. The crude mortality rates for cases and matched controls were $3.5 \%$ and $1.05 \%$, respectively. The mean length of stay significantly differed between the cases and matched controls (7.69 days vs 4.46 days), as did the mean cost of hospitalization ( $\$ 10,010$ vs $\$ 5,355)$ The extra length of hospital stay attributable to an ADE was $\$ 2,013$.

Both studies confirm that substantial costs of ADEs justify investment in effort to prevent these events.

FROM: 1. Bates DW, Spell N, Cullen DJ, Burdick E, Laird N, Peterson LA.. The cost of adverse drug events in hospitalized patients. JAMA 1997;277:307-311.

2. Classen DC, Pestonik SL, Evans RS, Lloyd JF, Burke JP. Adverse drug events in hospitalized patients. JAMA 1997;277:301-306.

\section{CDC's Immunization Practices Advisory Committee Revises Childhood Immunization Schedule}

The CDC's Immunization Practices Advisory Committee, the American Academy of Pediatrics, and the American Academy of Family Physicians recently changed some recommendations for childhood vaccination.

Poliovirus vaccines. The recommended age has been changed for administration of the third dose of inactivated poliovirus vaccine (IPV) in an all-IPV schedule to 6 to 18 months. The recommended ages for administration of polio vaccine in either an all-oral poliovirus vaccine or an all-IPV schedule are now the same (2, 4, 6-18 months, and 4-6 years).

Measles-mumps-rubella. The recommended age for the second dose of measles-mumps-rubella vaccine is now 4 to 6 years.

Hib Vaccines. Three Haemophilus influenzae type b (Hib) vaccines are licensed for infant immunization: (1) oligosaccharide conjugate Hib vaccine HibTITER (WyethLederle Laboratories, Pearl River, NY), (2) polyribosylribitol phosphate-tetanus toxoid conjugate (ActHIB and Omni-HIB, manufactured by Pasteur Merieux Connaught, Lyon, France, and distributed by Pasteur Merieux Connaught-USA, Swiftwater, PA, and SmithKline Beecham Pharmaceuticals, Philadelphia, PA), and (3) Haemophilus b conjugate vaccine (meningococcal protein conjugate; PRP-OMP; PedvaxHIB, Merck, Inc, West Point, $\mathrm{PA})$. These products now are considered interchangeable for primary, as well as booster, vaccination. If PRP-OMP is administered in a series with one of the other two products licensed for infants, the recommended number of doses to complete the series is determined by the other product (and not by the PRP-OMP). For example, if PRP-OMP is administered for the first dose at age 2 months and another vaccine is administered at age 4 months, a third dose of any of the three licensed Hib vaccines is recommended at age 6 months to complete the primary vaccine series.
Hepatitis B vaccine. For children born to hepatitis B surface antigen-negative mothers, the third dose of HBV should be administered at least 2 months after the second dose, but not before age 6 months. This represents a clarification in wording of the recommendations.

Other vaccines. The routine visit to the healthcare provider for adolescents aged 11 to 12 years remains an important time to ensure receipt of two doses of measlesmumps-rubella vaccine beginning at or after age 12months, one dose of varicella vaccine, and initiation or completion of the HBV vaccine series. Diphtheria and tetanus toxoid boosters still need to be administered routinely to all children at this age.

FROM: Centers for Disease Control and Prevention. Recommended childhood immunization schedule, United States, 1998. MMWR 1998;47(1):8-12.

\section{Guidelines for Antiretrovirals in Pediatric HIV}

The US Health Resources and Services Administration recently announced the availability of "Guidelines for the Use of Antiretroviral Agents in Pediatric HIV Infection." This document may be obtained from the National AIDS Clearinghouse at 1-800-458-5231, or at http://www.cdcnac.org, or from the HIV/AIDS Treatment Information Service at $800-448-0440$ or http://www. hivatis.org.

\section{Typing of Nocardia farcinica}

German investigators from the Institute for Medicine, Microbiology, and Immunology at the University of Bonn conducted a study on severe postoperative wound infections caused by Nocardia farcinica, observed repeatedly in a German hospital surgical ward. A pulsed-field gel electrophoresis protocol was established to characterize the genetic relatedness of the bacterial isolates from these infections. All 18 isolates from postoperative infections that have occurred since 1985 belong to a common endemic genotype; organisms of this genotype also were detected in the air of two rooms of the department where these postoperative infections occurred. In contrast, two environmental isolates from another building on the same campus showed a distinct genotype. Three cases of pulmonary infections, at a department that is located in proximity to the surgical department, also were caused by the endemic type, which suggests aerogenic spread of the endemic strain to these patients. Controls consisting of epidemiologically unrelated isolates from sporadic infections in other towns belonged in each case to a different genotype. Pulsed-field gel electrophoresis was shown to be suited well to differentiate various types of $N$ farcinica and revealed an endemic strain causing postoperative wound infections possibly after aerogenic transmission.

FROM: Blumel J, Blumel E, Yassin AF, Schmidt-Rotte $\mathrm{H}$, Schaal KP. Typing of Nocardia farcinica by pulsed-field gel electrophoresis reveals an endemic strain as source of hospital infections. J Clin Microbiol 1998;36:118-122. 